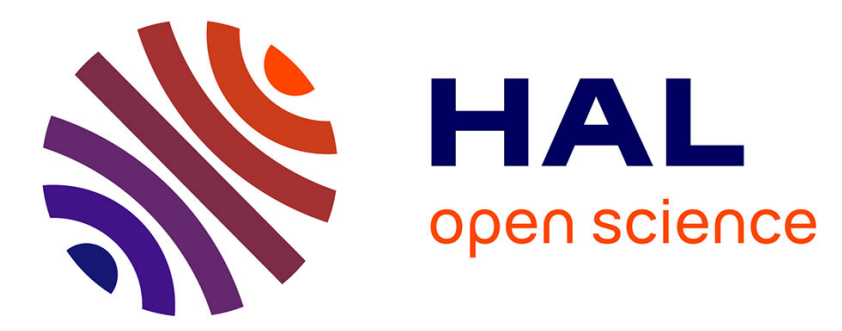

\title{
Development and Force/Position control of a new Hybrid Thermo-Piezoelectric MicroGripper dedicated to micromanipulation tasks.
}

Micky Rakotondrabe, Ioan Alexandru Ivan

\section{- To cite this version:}

Micky Rakotondrabe, Ioan Alexandru Ivan. Development and Force/Position control of a new Hybrid Thermo-Piezoelectric MicroGripper dedicated to micromanipulation tasks.. IEEE Transactions on Automation Science and Engineering, 2011, 8 (4), pp.824-834. 10.1109/TASE.2011.2157683 . hal00635587

\section{HAL Id: hal-00635587 \\ https://hal.science/hal-00635587}

Submitted on 25 Oct 2011

HAL is a multi-disciplinary open access archive for the deposit and dissemination of scientific research documents, whether they are published or not. The documents may come from teaching and research institutions in France or abroad, or from public or private research centers.
L'archive ouverte pluridisciplinaire HAL, est destinée au dépôt et à la diffusion de documents scientifiques de niveau recherche, publiés ou non, émanant des établissements d'enseignement et de recherche français ou étrangers, des laboratoires publics ou privés. 


\title{
Development and Force/Position Control of a New Hybrid Thermo-Piezoelectric MicroGripper Dedicated to Micromanipulation Tasks
}

\author{
Micky Rakotondrabe, Member, IEEE, and Ioan Alexandru Ivan, Member, IEEE
}

\begin{abstract}
A new microgripper dedicated to micromanipulation and microassembly tasks is presented in this paper. Based on a new actuator, called thermo-piezoelectric actuator, the microgripper presents both a high range and a high positioning resolution. The principle of the microgripper is based on the combination of the thermal actuation (for the coarse positioning) and the piezoelectric actuation (for the fine positioning). In order to improve the performances of the microgripper, its actuators are modeled and a control law for both the position and the manipulation force is synthesized afterwards. A new control scheme adapted for the actuators of the hybrid thermo-piezoelectric microgripper is therefore proposed. To prove the interest of the developed microgripper and of the proposed control scheme, the control of a pick-and-release task using this microgripper is carried out. The experimental results confirm their efficiency and demonstrate that the new microgripper and the control law are well suited for micromanipulation and microassembly applications.
\end{abstract}

Note to Practitioners-The works presented in this paper are motivated by the need of high performances systems dedicated to pick-transport-and-place tasks and their control. These tasks are one of the main base of the microassembly and micromanipulation applications. While pick-and-place tasks were successfully performed with classical microgrippers, they were very rarely automated. This is mainly due to the fact that automation in the micro/nanoworld is a new issue and the litterature only concerns the local control of microactuators and microrobots for the moment. Furthermore, existing microgrippers are limited to small range of positioning, making them hardly adapted for pick-and-place for large distance.

The core of this paper is to fulfil the requirement of high range, high resolution, and controlled microgrippers. For that, a microgripper based on a combination of the thermal and the piezoelectric actuations is developed. To improve the positioning accuracy and to control the manipulation force during a pick-and-place task, a new control scheme adapted for the actuators of the new microgripper is proposed. It is noticed that the proposed control scheme can also be applied to classical microgrippers as it is a generalization.

Manuscript received April 19, 2010; revised December 20, 2010 and April 10, 2011; accepted May 16, 2011. Date of publication June 13, 2011; date of current version October 05, 2011. This paper was recommended for publication by Associate Editor S. Fatikow and Editor K. Goldberg upon evaluation of the reviewers' comments. This work was supported in part by the EU FP7SP3People Program under Grant PIEF-GA-2008-219412 (New Micro-Robotic Systems featuring Piezoelectric Adaptive MicroStructures for Sensing and Actuating, with Associated Embedded Control: MicroPAdS).

M. Rakotondrabe is with the FEMTO-ST Institute, UMR CNRS 6174, UFC/ ENSMM/UTBM, Automatic Control and Micro-Mechatronic Systems Department, 25000 Besançon, France (e-mail: mrakoton@femto-st.fr).

I. A. Ivan is with the Valahia University of Târgoviste FIE, Târgoviste 130082, Romania.

Digital Object Identifier 10.1109/TASE.2011.2157683
Index Terms-Control, force and position, microassembly, microgripper, micromanipulation, modeling, pick-and-place, piezoelectric actuator, thermal actuator.

\section{INTRODUCTION}

$\mathbf{I}$ $\mathrm{N}$ microassembly, micromanipulation, and micrograsping applications, pick-and-place tasks are often practiced and constitute the primary base of operations. They consist in taking, transporting, and positioning small objects from one location to another. As the sizes of the objects to assemble or to manipulate are less than one millimeter, a pick-and-place task requires the use of systems that provide micrometric or submicrometric resolution and accuracy. This is why smart materials are often preferred relative to DC-motors and hinges for the development of microactuators, microrobots, and microsystems [1]. Indeed, smart materials propose less mechanical clearances than DC-motors and hinges and, therefore, propose more accuracy than them. Furthermore, smart materials allow more compact design because it is possible to design the systems with only one bulk material. Such compact design is essential for developing micro or nanorobots dedicated to work at the micro and nanoscale [2], [3]. For instance, it was demonstrated the possibility to obtain multiple degrees of freedom (dof) robots with millimetric sizes and nanometric resolution by using piezoelectric materials [4]. In [5], electroactive polymers have been presented as one of convenient materials for the design of small actuators in medical applications. Alternately, shape memory alloy can be used to develop a millimetric micromanipulator [6]. Finally, a packaged and accurate microsystem used to orient micro-mirror has been developed with the thermal bimetal principle in [7].

One of the well-known devices used for pick-and-place tasks in micromanipulation and microassembly is the microgripper. A microgripper is composed of two cantilevers which are single or both actuated by smart materials. Piezoelectric materials are the most employed to actuate microgrippers. Such a recognition is mainly due to their high resolution, high speed, and high force density. For instance, Haddab et al. [8] proposed a piezoelectric microgripper offering some tens of micrometers of positioning range and some milliseconds of settling time. Menciassi et al. [9] presented a piezoelectric microgripper with force sensor that was used for a force controlled manipulation [10]. Kemper [11] proposed a microgripper equipped with force sensor and actuated by piezoelectric stacks. In [12], a pneumatic microgripper 
was proposed. Finally, a microgripper can also be actuated with stick-slip motion principle such as in [13].

Shape Memory Alloy (SMA) has also been used to develop microgrippers with integrated force sensors [14]. Its main advantage relative to the piezoelectric design is the high range of positioning (more than the hundred of micrometers) and of force (over ten of millinewton). However, SMA materials have a stronger nonlinearity than piezoelectric ones, making them hard to control. In [15] and [16], electrostatic microgrippers able to precisely manipulate DNA and biological cells were proposed. The main advantage of electrostatic actuators is the high resonant frequency allowing a high bandwidth. However, the range of displacement is limited to some micrometers. More recently, electrothermal actuation has been used to develop force sensorized microgrippers [17], [18]. Like piezoelectric and electrostatic actuation, electrothermal has the advantage of using electrical energy for control, making the control setup easily available. In [19], Greitmann and Buser developed a microgripper based on the thermal bimorph principle. Thanks to the thermal bimorph actuation, each cantilever of the microgripper offers a large range of positioning (more than two hundreds of micrometers). However, it is more difficult to control the temperature of thermal actuators which often results a bad accuracy of the microgripper. To sum up, designing microgrippers having at the same time the high resolution, the high range, the convenient compactness, and the ease of control is a challenge. Microgrippers with such capabilities are, however, essential in large number of applications, particularly in pick-andplace tasks. The first motivation of this paper is to cover such a requirement by proposing a new kind of microgripper able to furnish nanometric resolution as well as a large range of displacement.

In the first part of this paper, the development of the new microgripper which is able to simultaneously perform a high range and a high resolution of positioning is presented. The developed microgripper is based on two cantilevers (actuators) presented in a previous work [20]. The principle of each cantilever is based on the thermal bimorph functioning combined with the piezoelectric effect. The cantilever is called hybrid thermo-piezoelectric actuator. The developed microgripper is therefore called hybrid thermo-piezoelectric microgripper. To actuate the thermal functioning, a Peltier module is used. It converts an electrical current into a heat flow. The main advantages of the proposed Peltier module are the use of electrical control signal and its embeddability. Thermal actuation is known to possess a low natural bandwidth, but when closed-loop with a feedback controller, their performances can fulfill the requirements in micromanipulation and microassembly contexts.

In the second part of this paper, the control of the developed microgripper is performed. Automation of systems in micromanipulation and microassembly applications is a recent issue. Many works on the microgrippers control are partial, i.e., only one cantilever is controlled. For instance, [10] and [18] present the force control, while Haddab [21] presents the displacement control, both using one cantilever of the microgripper. In our previous work [22], the complete control of both position and force was presented. However, the technique cannot be applied to a high range and high-resolution positioning system. In this paper, we propose a new control scheme adapted to such systems, and therefore adapted to the developed microgripper. The control of a pick-and-place task, with force and position control, is the final objective of this paper. Experimental results were performed and prove the efficiency of the proposed techniques.

This paper is organized as follows. Section II presents the development of the hybrid thermo-piezoelectric microgripper. Section III is devoted to the position control of one cantilever. In Section IV, the force control of the second cantilever is detailed. Finally, Section V concerns the complete control of the microgripper with an application of controlled pick-and-place task.

\section{Development of A New Hybrid THERMO-PIEZOELECTRIC MICROGRIPPER}

The principle and the development of the new microgripper are detailed in this section. In order to reach the high range and high resolution, the thermo-piezoelectric actuator presented in the previous work [20] is used for each cantilever. The principle of one hybrid thermo-piezoelectric cantilever is, therefore, introduced. Afterwards, the developed hybrid thermo-piezoelectric microgripper is performed.

\section{A. Presentation of the Hybrid Thermo-Piezoelectric Cantilever}

There are several choices to obtain a high range and a highresolution micropositioning system. Stepper motors (stick-slip and inch-worm) theoretically propose an unlimited range but their design is very complex and delicate because of the number of elements to be integrated in the motors. The combination of a high-range system (DC-motors) with a fine positioning system is also an alternate possibility. However, this approach leads to greater sizes of the whole system. In addition, this approach proposes a lower accuracy due to the clearance and/or the eventual bad alignment of the assembled elementary systems. In [20], a compact design of microactuator was proposed. It is actuated in two ways: thermal actuation for the high range, and piezoelectric actuation for the high resolution. The actuator is composed of a unimorph piezoelectric cantilever, made up of one piezolayer (lead zirconate titanate or PZT ceramics) and a passive layer (nickel). When a voltage is applied to the PZTlayer, the cantilever bends accordingly to the converse piezoelectric effect. On the other hand, when the cantilever is submitted to a temperature variation, a bending is also obtained as the unimorph is equivalent to a thermal bimetal. To facilitate the thermal actuation, the cantilever is embedded to a Peltier model or TEC-device (Thermo-Electric-Cooler). The main advantage of the TEC-device is the easiness of control: use of electrical current excitation, and possibility to reverse the heating into cooling so that both positive and negative directions of the bending are possible. One face of the TEC-device is connected to the cantilever while the second face is embedded on a cooling block. Fig. 1(a) and (b) presents the CAD-drawing of the hybrid cantilever and a photo, respectively. The resolution of the actuator is submicrometric and the range can reach over two hundreds of micrometers when applying the maximal input control. In order to avoid the depolarization of the piezoelectric material, however, the absolute temperature should be widely lower than its Curie temperature during the thermal actuation. The Curie 


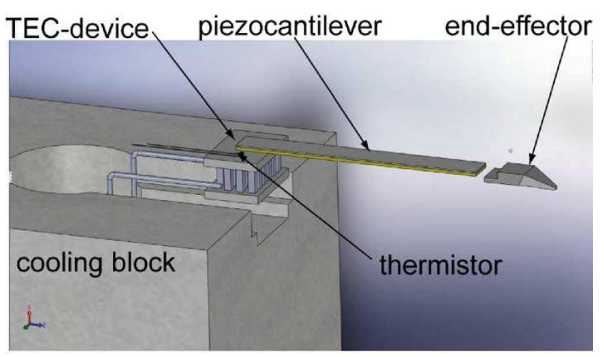

(a)

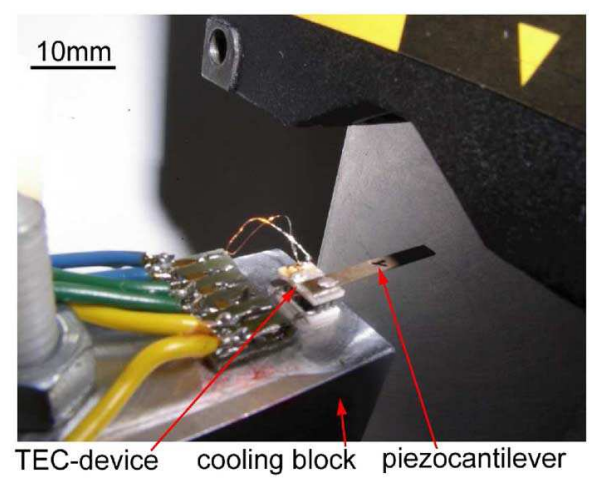

(b)

Fig. 1. (a) CAD-drawing and (b) a photograph of the thermo-piezoelectric cantilever.

temperature is above $350{ }^{\circ} \mathrm{C}$ for PZT ceramics. To have a sufficient security, the working absolute temperature is limited to be lower than $50{ }^{\circ} \mathrm{C}$ (differential temperature $\Delta T$ lower than $35^{\circ} \mathrm{C}$ ). The positioning provided by such temperature (up to $100 \mu \mathrm{m}$ ) is sufficient for the considered micromanipulation/microassembly contexts. On the other hand, the maximal voltage applicable to the PZT-layer is $100 \mathrm{~V}$. Above this value, there is a risk of damage of the actuator. Therefore, a working voltage lower than $60 \mathrm{~V}$ is chosen for all experiments. Such an input corresponds to nearly $25 \mu \mathrm{m}$.

\section{B. Prototype of the Hybrid Thermo-Piezoelectric Microgripper}

The developed thermo-piezoelectric microgripper are based on two thermo-piezoelectric cantilevers. Each of the cantilever has a TEC-device with its cooling block. A PCB (printed circuit board) layer is used to separate and impose the gap between the two cantilevers [Fig. 2(a)]. This PCB is also used to conduct the different elctrical circuits from the two piezocantilevers and the two TEC-devices to the connectors. Finally, the PCB allows an ease of mounting and a packaging of the microgripper. Fig. 2(b) presents a photograph of the prototype equipped with end-effectors. The sizes of the piezocantilevers are: $15 \mathrm{~mm} \times 2 \mathrm{~mm} \times 0.3 \mathrm{~mm}$ in which the PZT-layer has a thickness of $0.2 \mathrm{~mm}$. The two cooling blocks, which at the same time constitute the support, characterize the maximal sizes of the whole system. In the prototype, the dimensions of each cooling block are $20 \mathrm{~mm} \times 25 \mathrm{~mm} \times 30 \mathrm{~mm}$.

\section{Governing Equations of the Thermo-Hybrid Microgripper}

Consider the unimorph microgripper manipulating a small object as depicted in Fig. 3. In Fig. 3, we have:

- $\delta_{l o}$ (resp. $\delta_{\text {ro }}$ ) as the distance between the left (resp. right) piezocantilever and the object before contact (at rest);
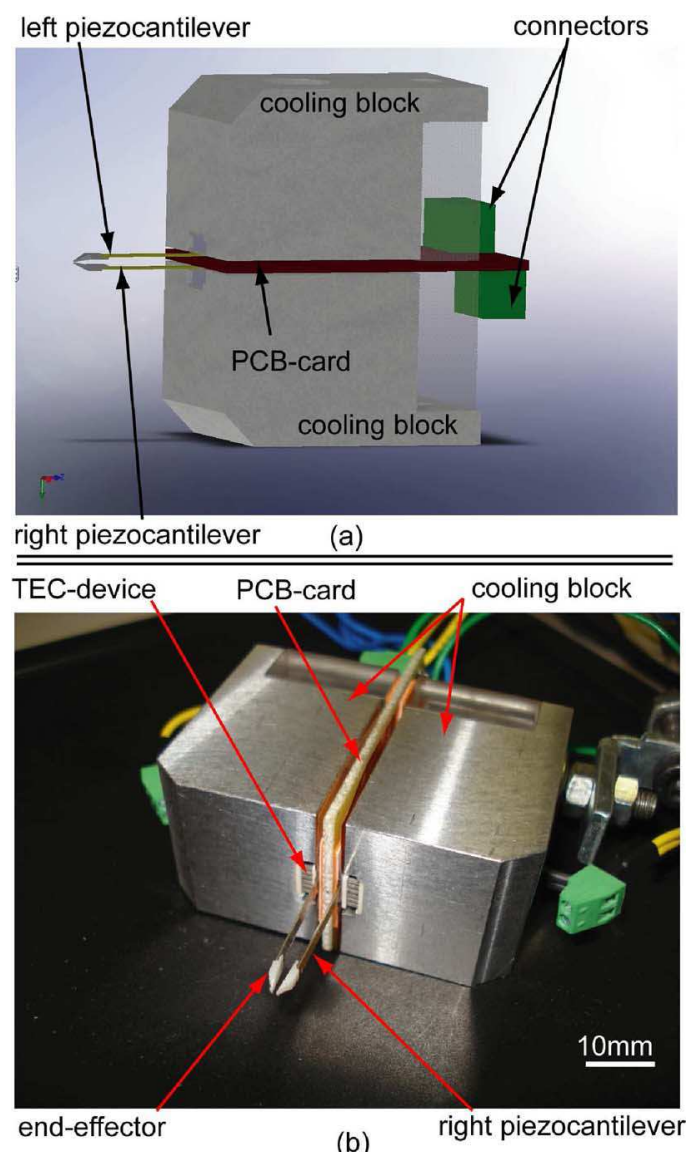

Fig. 2. (a) CAD-drawing and (b) a photograph of the hybrid thermo-piezoelectric microgripper [20].

- $\delta_{l}$ as the deflection of the left piezocantilever when bent by the thermal actuation and/or by the piezoelectric actuation, while $\delta_{r}$ for the right piezocantilever;

- $F_{m}$ is the manipulation force, i.e., force applied by the left piezocantilever to the object. It is possible to consider the force applied by the right actuator by changing the signs of the variables. However, in the sequel, the left piezocantilever is considered as the force actuator.

1) Model of One Piezocantilever: Smits and Choi [23] gave an analytical relation between the output deflection $\delta$, the input voltage $U$ and the external force $F_{x}$ applied at the tip of a unimorph piezocantilever as

$$
\delta=d \cdot U+c \cdot F_{x}
$$

where $d$ is the piezoelectric constant and $c$ the elastic constant.

To extend the static model (1) into a dynamic and complete model, it has been admitted that both the mechanical term and the piezoelectric term has the same dynamics [24]. Denoting $D_{U}(s)$ (such as $\left(D_{U}(0)=1\right.$, i.e., $D_{U}$ is a normalized dynamic term) the term that models the dynamics of the piezoelectric microactuator, we have

$$
\delta=d \cdot D_{U}(s) \cdot U+c \cdot D_{U}(s) \cdot F_{x} .
$$

Finally, [20] shows that the dynamic model between the input current $I$ applied to a TEC-device and the output deflection of a thermo-hybrid cantilever is

$$
\delta=f \cdot D_{I}(s) \cdot I
$$




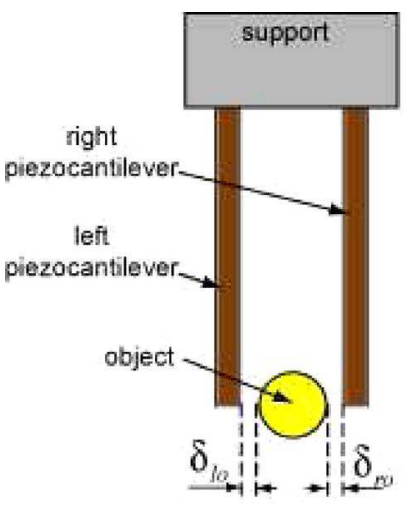

(a)

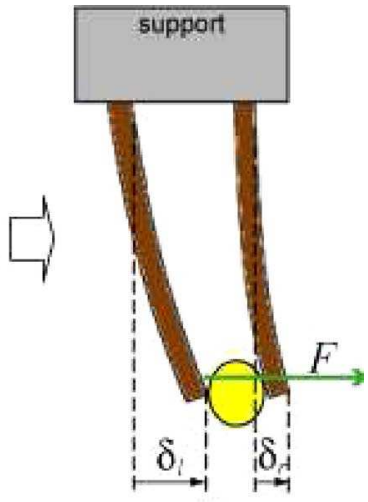

(b) positive direction for the displacement/force

Fig. 3. Schematic of a microgripper manipulating a small object.

where $f$ is a coefficient that lumps together the TEC-device and the thermal bimorph element characteristics. $D_{I}(s)$ (such as $D_{I}(0)=1$ ) is the normalized dynamic part.

Knowing that the thermal effect is affine in the piezoelectric model [25], the thermal contribution in (3) is inserted into (2) in order to derive the constitutive model of a thermo-hybrid cantilever

$$
\delta=d_{i} \cdot D_{U i}(s) \cdot U i+c_{i} \cdot D_{U i}(s) \cdot F_{i}+f_{i} \cdot D_{I i}(s) \cdot I_{i}
$$

where $i \in\{l, r\}$ denotes the left or the right piezocantilever.

2) Deformation Model of the Manipulated Object: Consider again Fig. 3(b). The deformation of the manipulated object can be written as follows:

$$
\left(\delta_{l}-\delta_{l o}\right)-\left(\delta_{r}-\delta_{r o}\right)=c_{o} \cdot F
$$

where $c_{o}=(1) /\left(k_{o}\right)$ is the compliance of the object, $k_{O}$ being its stiffness. The manipulation force is $F=-F_{l}, F_{l}$ being the reaction force applied by the object to the left cantilever. As we can see, the deformation model of the object is static (spring). This can be assumed as long as the mass is negligible, which is the case for micro-objects and microcomponents.

3) Governing Equations of the Microgripper: Finally, using (4) and (5), the governing equations of the thermo-hybrid microgrippers are derived

$\left\{\begin{array}{l}\delta_{l}=d_{l} \cdot D_{U l}(s) \cdot U_{l}-c_{l} \cdot D_{U l}(s) \cdot F+f_{l} \cdot D_{I l}(s) \cdot I_{l} \\ \delta_{r}=d_{r} \cdot D_{U r}(s) \cdot U_{r}+c_{r} \cdot D_{U r}(s) \cdot F+f_{r} \cdot D_{I r}(s) \cdot I_{r} \\ \left(\delta_{l}-\delta_{r}\right)-\Delta \delta_{o}=c_{o} \cdot F\end{array}\right.$

with $\Delta \delta_{o}=\delta_{l o}-\delta_{\text {ro }}$.

In the next two sections, the control of each hybrid thermopiezoelectric cantilever is considered: the position (deflection) control for the right hybrid cantilever, while the force control for the left one.

\section{Position Control of the Right Hybrid CANTILEVER}

In this section, the position control scheme for the right hybrid thermo-piezoelectric cantilever is considered. The aim is to improve its performances in order to adapt them to the requirements of pick-and-place tasks in the microworld. For instance, the positioning or assembly of a micro-object takes two steps.

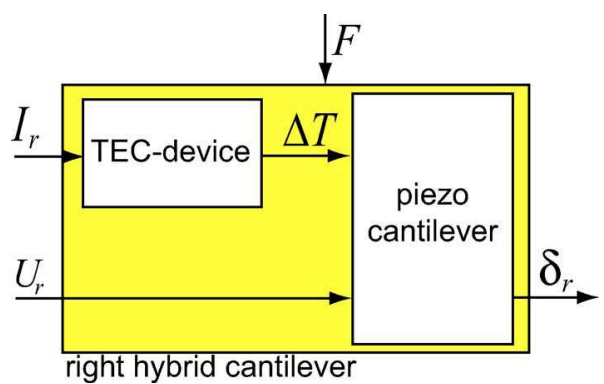

Fig. 4. Systemic scheme of the right hybrid cantilever.

- A coarse positioning which consists to position the object at a long distance without a special accuracy. The electro-thermomechanical functioning of the hybrid cantilever can be used for that. We mean by electro-thermomechanical functioning the actuation from the electrical current $I_{i}$, applied to the TEC-device, to the bending obtained by the thermal bimetal principle.

- A fine positioning that can be obtained using the piezoelectric functioning of the hybrid cantilever.

To manage the two positioning steps, a simple and effective hybrid control law that makes possible the automatic switching between the coarse and fine positioning modes is proposed. Here, hybrid control means that continuous and discrete aspects are present. The discrete states are composed of the two positioning modes.

After presenting the modeling and identification aspects, a dynamic (continuous) controller for each mode is first designed. Finally, the complete control that includes the switching between the two modes is detailed.

\section{A. Modeling}

The model is given by the second equation of (6) and the systemic scheme is presented by Fig. 4 . While the output is the deflection $\delta_{r}$, there are two input controls: the voltage $U_{r}$ and the electrical current $I_{r}$. The force applied by the object to the cantilever denoted by $F_{r}=F$ is a disturbance to be rejected by the controller.

\section{B. Identification}

1) Electro-Thermomechanical Part: First, let us identify the parameters of the electro-thermomechanical $f_{r} \cdot D_{I r}(s) \cdot I_{r}$. For that, a step input $I_{r}=0.2 \mathrm{~A}$ is applied and $U_{r}$ is left equal to zero. The step response which corresponds to the behavior of a first order system is easily identified using a trial-error adjustment. While the identified model is given by (7), Fig. 5 shows the model simulation which well fits to the experimental result

$$
G_{r}^{I \rightarrow \delta}=\frac{\delta_{r}}{I_{r}}=\frac{400}{16 s+1}
$$

such as $f_{r}=400[(\mu m) /(A)]$

2) Piezoelectric Part: To identify the parameters of the piezoelectric part $\delta_{r}=d_{r} \cdot D_{U r}(s) \cdot U_{r}$, a harmonic analysis is applied. Harmonic analysis provides more accurately identified parameters (damping coefficient, natural frequency) relative to a step response analysis if the system is resonant. For the identification process, a sine input voltage $U_{r}$ of amplitude $20[\mathrm{~V}]$ and a frequency ranging between $80[(\mathrm{rad}) /(s)]$ and 


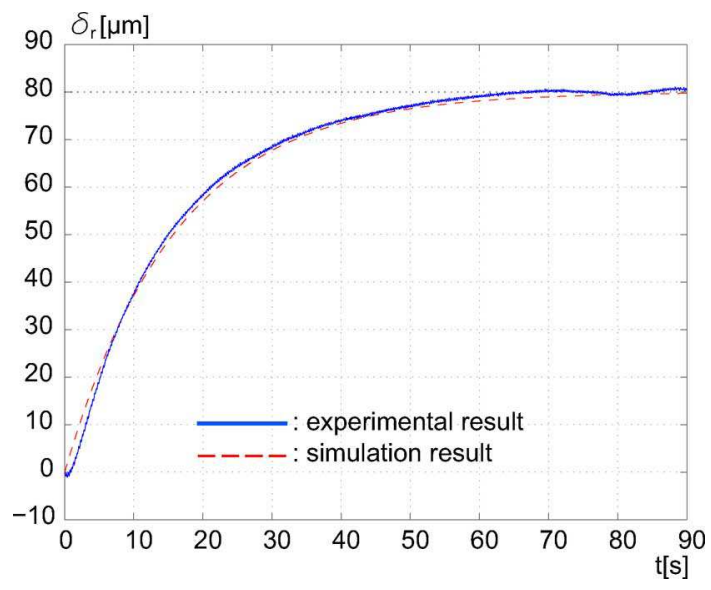

Fig. 5. Step response of the electro-thermomechanical part with $I_{r}=0.2[A]$ : experimental result and model simulation.

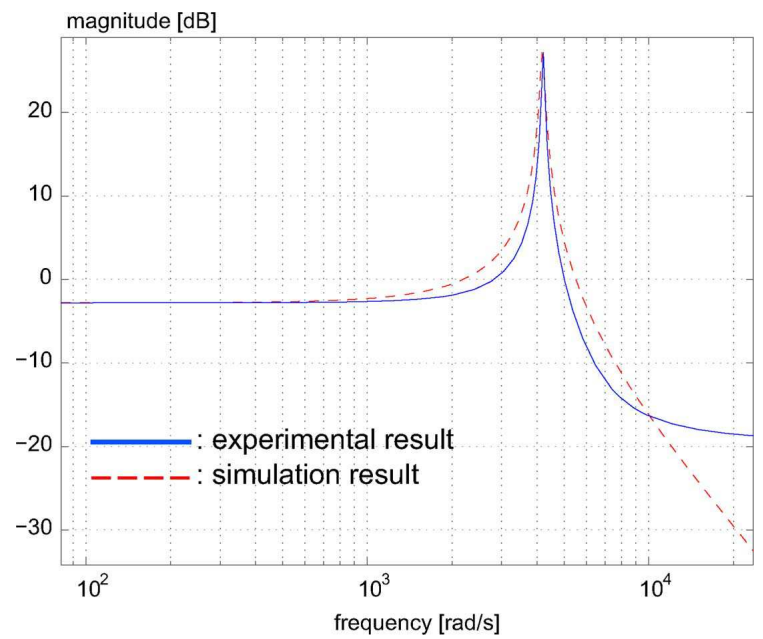

Fig. 6. Harmonic response of the piezoelectric part: experimental result and model simulation.

$11[(\mathrm{krad}) /(s)]$ is used. The model parameters, considered to be a second order, are derived using a trial-error adjustment. Eq. (8) gives the model. Fig. 6 depicts the model simulation compared to the experimental result and shows that it is accurate enough

$$
G_{r}^{U \rightarrow \delta}=\frac{\delta_{r}}{U_{r}}==\frac{0.725}{5.7 \times 10^{-8} s^{2}+7.2 \times 10^{-6} s+1}
$$

such as $d_{r}=0.725[(\mu m) /(V)]$.

\section{Coarse Positioning Control Through the Electro-Thermomechanical Functioning}

In this section, the electro-thermomechanical functioning is controlled. Fig. 7(a) gives the closed-loop scheme, where $\delta_{r}^{\text {ref }}$ is the reference input. A Proportional-Integral (PI) structure $C_{I r}=\left(I_{r}\right) /\left(\varepsilon_{r}\right)=K_{p r}\left(1+\left(K_{i r}\right) /(s)\right)$ is proposed. The PI structure is enough to improve the dynamics of the electro-thermomechanical functioning as well as to make the actuator converge towards the reference input. The controller gains were adjusted using the Ziegler-Nichols rules, whose resulting performances were sufficient for the used application. We have $K_{p r}=0.005$ and $K_{i r}=0.065$.

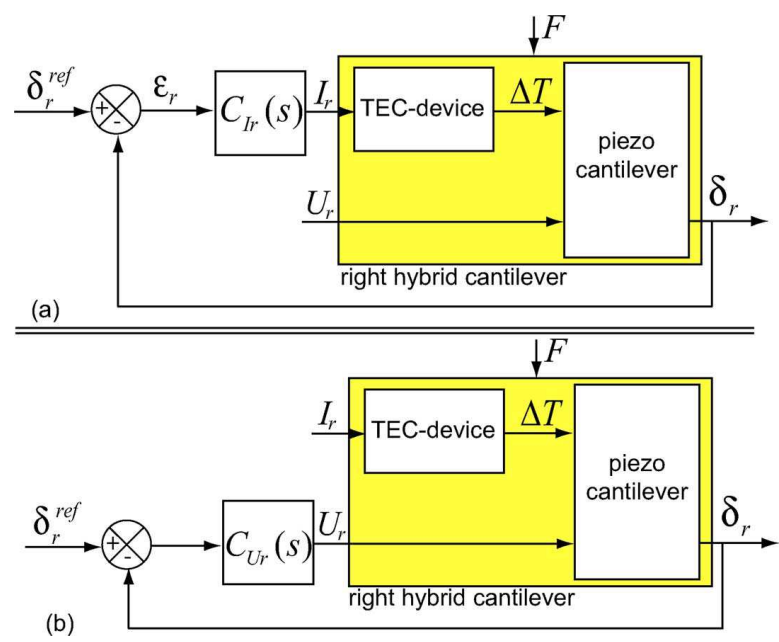

Fig. 7. Closed-loop scheme (a) for the coarse positioning mode (electro-thermomechanical functioning) and (b) for the fine mode (piezoelectric functioning).

\section{Fine Positioning Control Through the Piezoelectric Functioning}

When the distance between the reference input $\delta_{r}^{\text {ref }}$ and the output $\delta_{r}$ becomes less than a defined valued $\delta_{r}^{\text {lcp }}$ (limit coarse positioning), the fine positioning mode is activated and the piezoelectric functioning is controlled. The constant value $\delta_{r}^{\text {lcp }}$ gives the maximal range within which the piezoelectric functioning works. It can be chosen using the applicable maximal voltage $U_{r}$. In our case, this limit is $60 \mathrm{~V}$, corresponding to a displacement of $20 \mu \mathrm{m}$. Fig. 7(b) shows the corresponding closed-loop scheme.

The control of the fine mode comes back to the control of a classic piezoelectric cantilever. This system is very resonant (see peak resonance in Fig. 6). Many works have been reported to control resonant piezoelectric cantilevers, but the $H_{\infty}$ control law was particularly appreciated for its efficiency to completely damp the resonance peaks and to account model uncertainty [1], [22]. This control design is therefore proposed to improve the performances of the fine positioning mode in our case. After computation, the following controller is obtained:

$$
C_{U r}(s)=\frac{-15810\left(s+4 \times 10^{6}\right)\left(s-1.4 \times 10^{4}\right)}{\left(s+1.5 \times 10^{7}\right)(s+0.3)} .
$$

\section{E. Complete Position Control of the Right Hybrid Thermo-Piezoelectric Cantilever}

To bring together the control of the two previous modes into one scheme, the scheme presented in Fig. 8 is proposed. The principle of the complete control is as follows.

- If the output $\delta_{r}$ is far away from the reference $\delta_{r}^{\text {ref }}$, i.e., $\left|\varepsilon_{r}\right|=\left|\delta_{r}^{\text {ref }}-\delta_{r}\right|>\delta_{r}^{\text {lcp }}$, then only the electro-thermomechanical functioning is controlled and the coarse positioning works.

- When the output $\delta_{r}$ is close to the reference, i.e., $\left|\varepsilon_{r}\right|=$ $\left|\delta_{r}^{\text {ref }}-\delta_{r}\right| \leq \delta_{r}^{\text {lcp }}$, then the discrete controller $\Sigma_{r}$ switches on the fine mode and the piezoelectric functioning becomes controlled. 


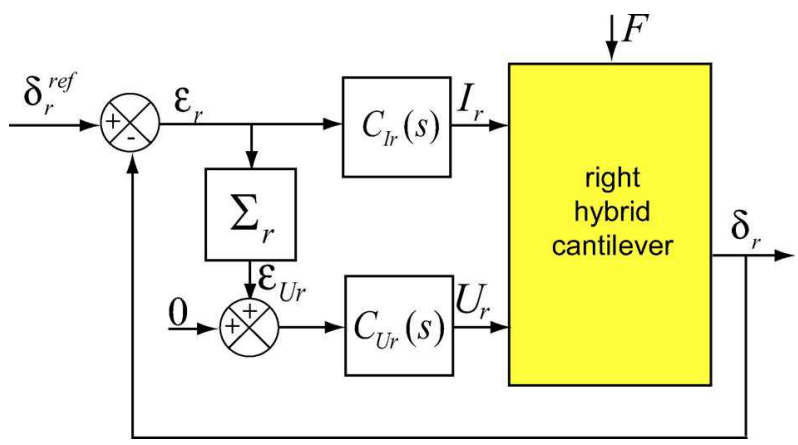

Fig. 8. Complete control scheme of the right hybrid cantilever.

The discrete controller $\Sigma_{r}$ is defined by

$$
\begin{aligned}
& \begin{array}{ll}
\Sigma_{r}: & \\
\text { if } & \left|\varepsilon_{r}\right|>\delta_{r}^{\text {lcp }} \\
& \varepsilon_{U r}=0 \\
\text { else } & \text { then } \\
\text { end } & \varepsilon_{U r}=\varepsilon_{r}
\end{array}
\end{aligned}
$$

The controllers as presented in Fig. 8 were implemented using the Simulink software in a computer-dSPACE real-time hardware with a sampling time $h=0.2 \mathrm{~ms}$. The position (deflection) of the cantilever is measured with an optical displacement sensor (from Keyence) having a range of $\pm 200 \mu \mathrm{m}$ and a resolution up to $10 \mathrm{~nm}$. For the experiments, a maximal fine positioning limit $\delta_{r}^{\mathrm{lcp}}=20 \mu \mathrm{m}$ is chosen. This choice satisfies the maximal voltage allowed for the actuator, as discussed in Section II-A. During the experiments, a series of reference input steps $\delta_{r}^{\text {ref }}$ were applied at different instants. Fig. 9(a) pictures how the output $\delta$ successfully reaches the reference. In order to see the two modes, the voltage $U_{r}$ and the current $I_{r}$ were also recorded [Fig. 9(b) and (c)]. Finally, Fig. 9(d) shows the temperature applied by the TEC-device to the right cantilever and measured with a thermistor. We can remark that when the distance between the reference and the output is more than $\delta_{r}^{\mathrm{lcp}}=20 \mu \mathrm{m}$, only the current $I_{r}$ changes. When this distance becomes less than $\delta_{r}^{\text {lcp }}$, the voltage $U_{r}$ starts to change, while $I_{r}$ becomes constant. In fact, the controller $C_{I r}(s)$ first provides a high value of current $I_{r}$ in order to accelerate the heating (or cooling) process and, therefore, to accelerate the coarse displacement. When the fine positioning starts functioning, the current decreases and becomes constant to hold the last value of the coarse position. Fig. 10 is a zoom of a step response for $\delta_{r}^{\text {ref }}=80 \mu \mathrm{m}$. It shows more clearly the switching from the coarse positioning to the fine positioning modes.

\section{Force Control of the Left Hybrid CANTILEVER}

This section is focused on the force control of the left hybrid thermo-piezoelectric cantilever. The process is similar to the position control of the right hybrid cantilever. After the modeling and identification procedure, the control of each mode (coarse and fine modes) is separately presented. Afterwards, the two controllers of the two modes are mixed into one scheme using a discrete controller.
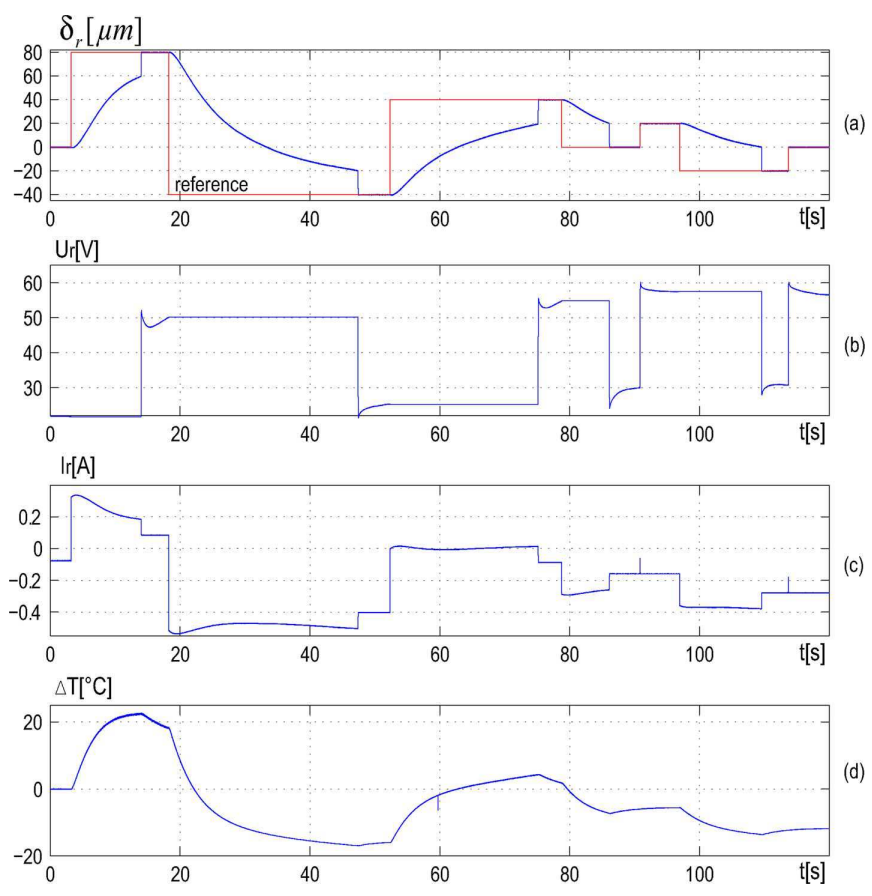

Fig. 9. Step response of the closed-loop hybrid cantilever: (a) reference and measured output deflection, (b) applied voltage, (c) applied electric current to the TEC-device, and (d) measured temperature difference.

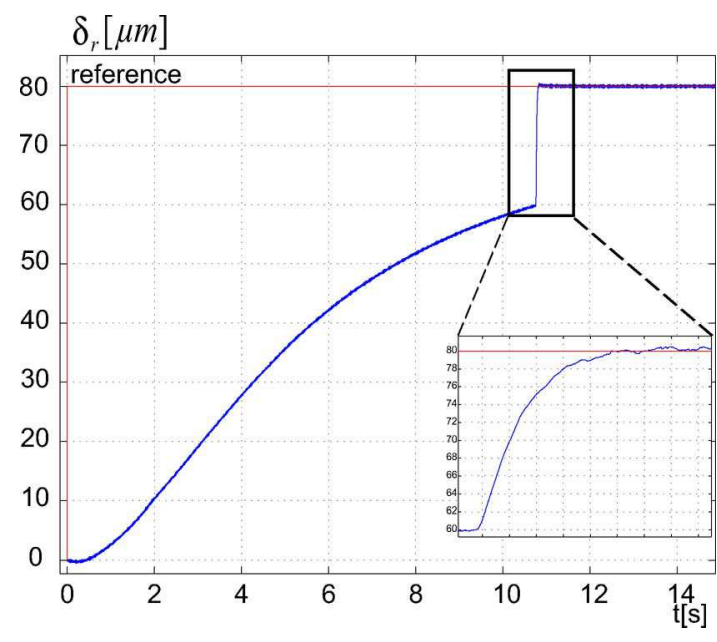

Fig. 10. Zoom of a step response of the closed-loop hybrid cantilever.

\section{A. Modeling}

From the first and the third equations of the model (6), the manipulation force is deduced

$$
\begin{aligned}
F=\frac{1}{\left(c_{o}+c_{l}\right)} D_{k l}(s)\left[d_{l} D_{U l}(s) U_{l}\right. & \\
& \left.+f_{l} D_{I l}(s) I_{l}-\left(\delta_{r}+\Delta \delta_{o}\right)\right]
\end{aligned}
$$

where $D_{k l}(s)=\left(\left(c_{o}+c_{l}\right)\right) /\left(\left(c_{o}+c_{l} D_{U l}(s)\right)\right)$ is a normalized dynamic part such as $D_{k l}(0)=1$.

As can be observed, the model is dependent on the compliance $c_{o}$ of the object. However, it is not convenient to identify $c_{o}$ at each change of manipulated object. Therefore, we propose to fix $c_{o}=0$ (corresponding to a rigid object with stiffness 


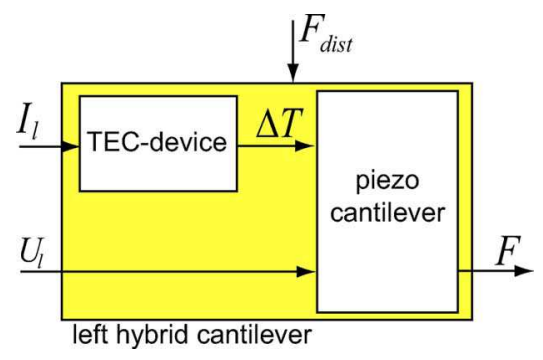

Fig. 11. Systemic scheme of the left hybrid cantilever.

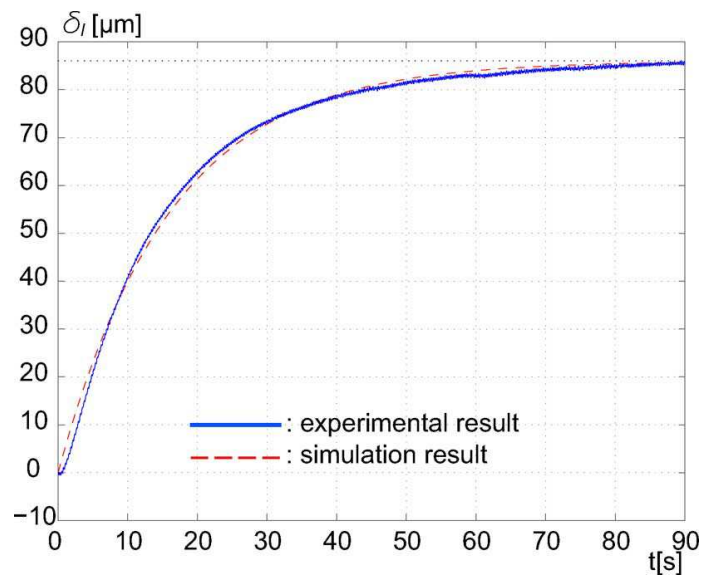

Fig. 12. Step response of the electro-thermomechanical part: experimental result and model simulation.

$\left.k_{o} \rightarrow \infty\right)$ in the used model and to synthesize a robust control law in order to ensure the stability for a large range of $c_{o}$. Furthermore, $D_{k l}(s)$ is set equal to one whatever $s$ is in order to simplify the model. This is possible if the control law is robust enough to reject uncertainty effects in high frequency [22]. The final model is therefore

$$
F=\frac{d_{l}}{c_{l}} D_{U l}(s) U_{l}+\frac{f_{l}}{c_{l}} D_{I l}(s) I_{l}+F_{\text {dist }}
$$

where the disturbance is $F_{\text {dist }}=-\left(\left(\delta_{r}+\Delta \delta_{o}\right)\right) /\left(c_{l}\right)$.

The systemic scheme corresponding to the model is presented in Fig. 11. While the output is the manipulation force $F$, the input controls are the voltage $U_{l}$ and the electrical current $I_{l}$. Finally, the disturbance $F_{\text {dist }}$ must be taken into account during the controller synthesis.

\section{B. Identification}

1) Electro-Thermomechanical Part: Here, the parameters $f_{l}$ and $D_{I l}$ are identified. For that, a step current input $I_{l}=0.2 \mathrm{~A}$ is applied to the hybrid cantilever and the output deflection $\delta_{l}$ is reported. It is noticed that no object is in contact with the cantilever during the identification process. The step response has a first-order behavior and is identified using a trial-error method. While the model simulation and the experimental results are pictured in Fig. 12, the model is given by

$$
G_{l}^{I \rightarrow \delta}=\frac{\delta_{l}}{I_{l}}=\frac{430}{16 s+1}
$$

such as $f_{l}=430[(\mu m) /(A)]$.

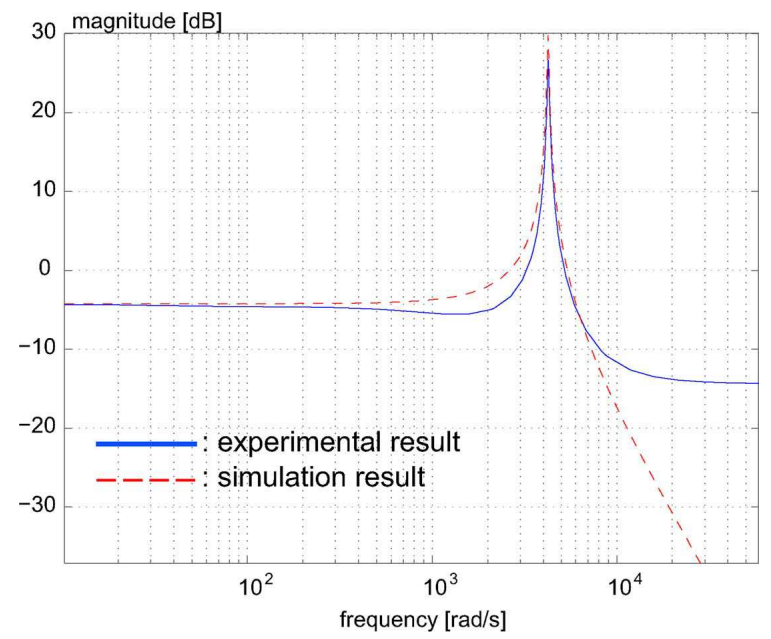

Fig. 13. Harmonic response of the piezoelectric part: experimental result and model simulation.

2) Piezoelectric Part: To identify the piezoelectric parameters $d_{l}$ and $D_{U l}$, a harmonic analysis is carried out. Once again, the object is not in contact with the cantilever. A sine input voltage with amplitude $20 \mathrm{~V}$ and frequency ranging between $80[(\mathrm{rad}) /(s)]$ and $11[(\mathrm{krad}) /(s)]$ is used. Using the trial-error tuning, a second-order model (see Fig. 13 for the comparison between the model simulation and the experiment) can be derived

$$
G_{l}^{U \rightarrow \delta}=\frac{\delta_{l}}{U_{l}}=\frac{0.615}{5.5 \times 10^{-8} s^{2}+4.7 \times 10^{-6} s+1}
$$

such as $d_{l}=0.615[(\mu m) /(V)]$

3) Identification of the Compliance $C_{l}$ : To identify the last parameter $c_{l}$ of (12), a weight with a known value $(m=1 \mathrm{~g})$ is put at the tip of the cantilever and the resulting deflection is measured: $\delta_{l}=20 \mu \mathrm{m}$. The compliance is afterwards calculated: $c_{l}=2[(\mu m) /(m N)]$.

\section{Coarse Mode Control Through the Electro-Thermomechanical Functioning}

To control the force for a large range mode, also called coarse mode, the electro-thermomechanical functioning should be activated. Fig. 14(a) gives the closed-loop scheme, where $F^{\text {ref }}$ is the reference input. As stated above, the used nominal model [see (12)] is very approximative because the object parameter $c_{o}$ and the high dynamics $D_{k}(s)$ were neglected. Therefore, a robust $H_{\infty}$ controller is used to maintain the stability for a large range of manipulated objects. After computation of the controller, we obtain

$$
C_{I l}(s)=\frac{0.15(s+30)(s+0.15)(s+0.063)}{(s+78)(s+3.2)(s+0.13)(s+0.0015)} .
$$

\section{Fine Mode Control Through the Piezoelectric Functioning}

When the error between the reference force and the manipulation force $\varepsilon_{F}=F^{\text {ref }}-F$ is less than a defined value denoted $F^{l c}$ (limit coarse mode), the fine mode starts to work. Like in the position control of the right cantilever, the fine mode is obtained by controlling the piezoelectric functioning. The corresponding closed-loop scheme is pictured in Fig. 14(b). The behavior of 

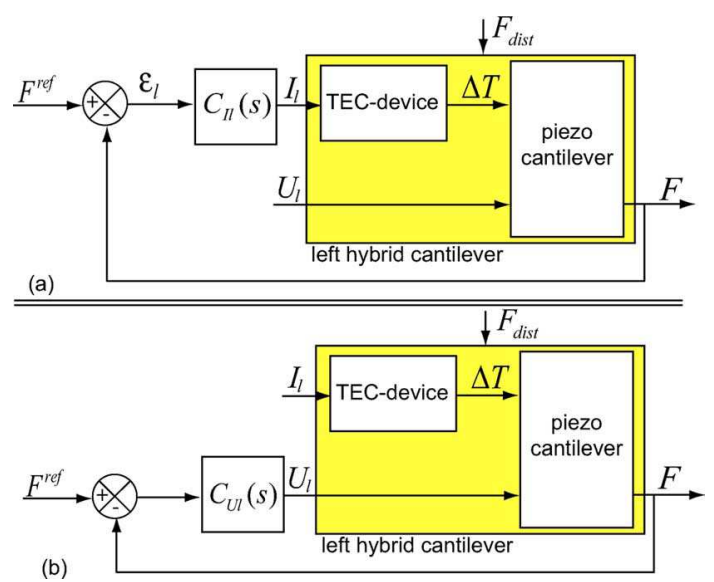

Fig. 14. Closed-loop scheme: (a) for the coarse mode and (b) for the fine mode.

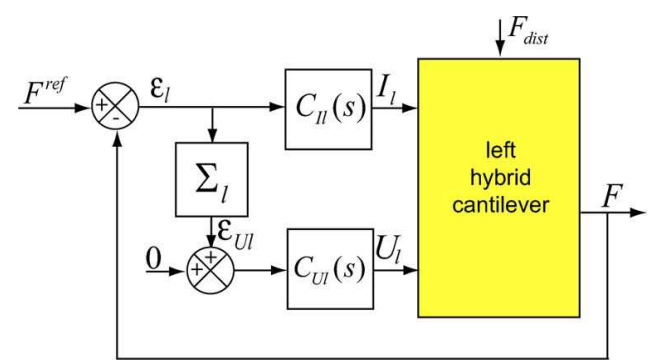

Fig. 15. Complete control scheme of the left hybrid cantilever.

the cantilever when actuated piezoelectrically is very resonant (see the resonant peak in Fig. 13). Furthermore, the model is subjected to uncertainty. Therefore, a $H_{\infty}$ control law is used to compute the corrector $C_{U l}$. It derives equation (16) shown at the bottom of the page.

\section{E. Complete Force Control of the Left Hybrid Thermo-Piezoelectric Cantilever}

Fig. 15 pictures the complete force control of the left hybrid thermo-piezoelectric cantilever. The discrete controller $\Sigma_{l}$ is used to switch between the coarse and the fine modes. This controller is defined by

$$
\begin{aligned}
& \Sigma_{l}:
\end{aligned}
$$

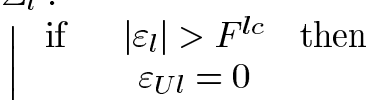

$$
\begin{aligned}
& \text { else } \\
& \varepsilon_{U l}=\varepsilon_{l} \\
& \text { end. }
\end{aligned}
$$

The controllers as presented in Fig. 15 were implemented. However, there exists no convenient microforce sensor to measure $F$ for the feedback. Thus, the deflection of the left hybrid cantilever is measured using an optical displacement sensor
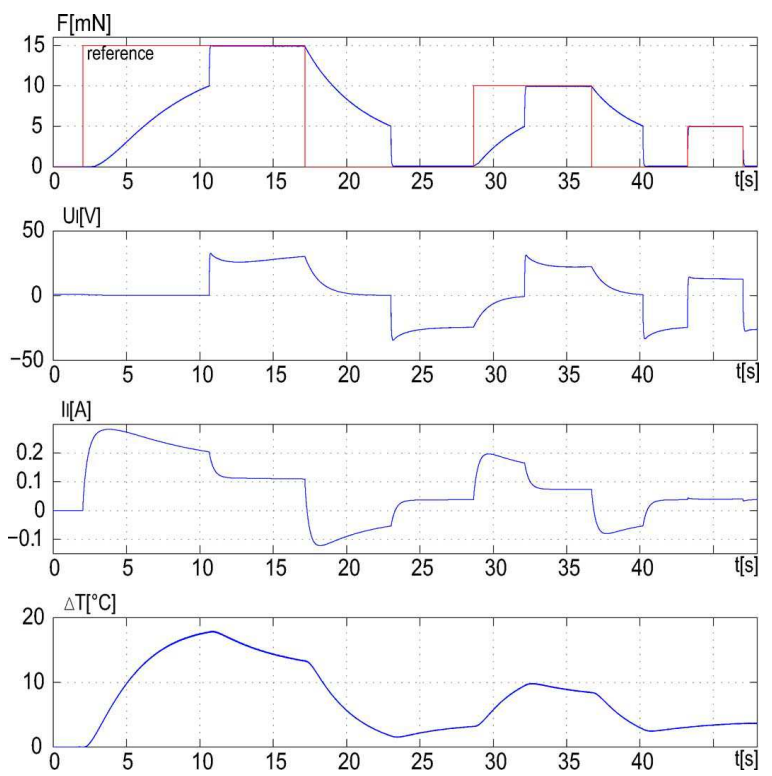

Fig. 16. Response of the closed-loop hybrid cantilever: (a) reference and measured output force, (b) applied voltage, (c) applied electric current to the TECdevice, and (d) measured temperature difference.

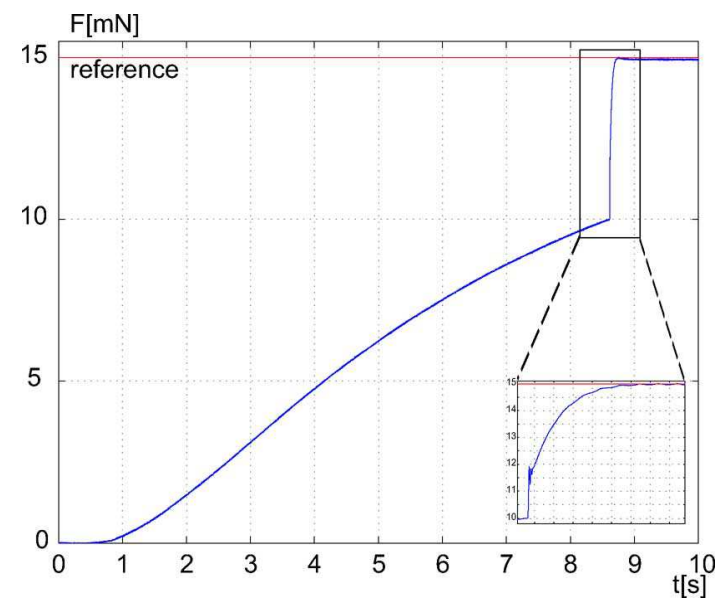

Fig. 17. Zoom on a stepResponse of the closed-loop hybrid cantilever.

similar to the right cantilever and an observer is used to provide an estimate of the force $F$. The displacement sensor (from Keyence) has a range of $\pm 200 \mu \mathrm{m}$ and a resolution up to $10 \mathrm{~nm}$. The force observer, based on a previous work [26], applies an inverse model to the output displacement to come back to the force. For the experiment, $F^{l c}=5$ is chosen to be $F^{l c}=5 \mathrm{mN}$. The experiments were performed using a series of reference steps $F^{\text {ref }}$. The results are presented in Fig. 16. They show that the output well tracks the input reference [Fig. 16(a)]. In Fig. 16(b), the activation of the fine mode is clearly shown (for example at $t=10.5 \mathrm{~s}$ ). Similarly, to the deflection control in Section III, the controller $C_{I l}(s)$ first provides a high value of current $I_{l}$ in order to speedup the heating (or cooling) process,

$$
C_{U l}(s)=\frac{10561(s+100)(s+20)\left(s^{2}+85 s+1.8 \times 10^{7}\right)}{(s+4161)(s+17)(s+1)\left(s^{2}+1468 s+1.5 \times 10^{7}\right)}
$$




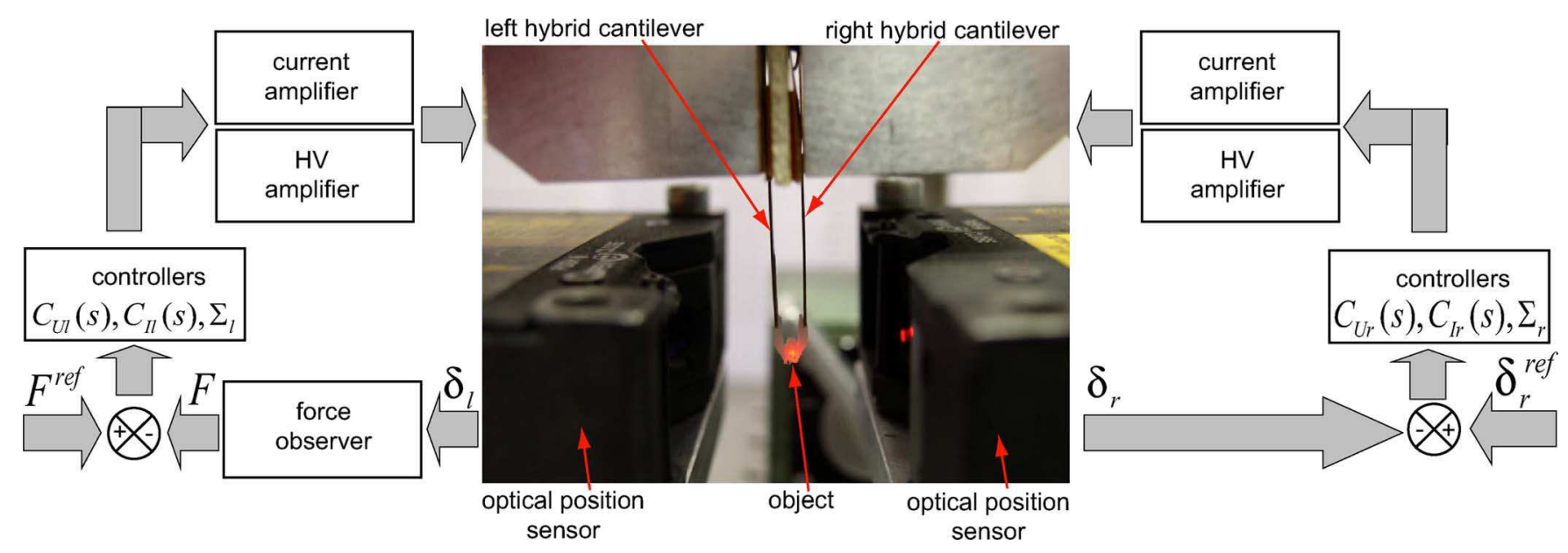

Fig. 18. Experimental setup used for the controlled pick-and-place task.

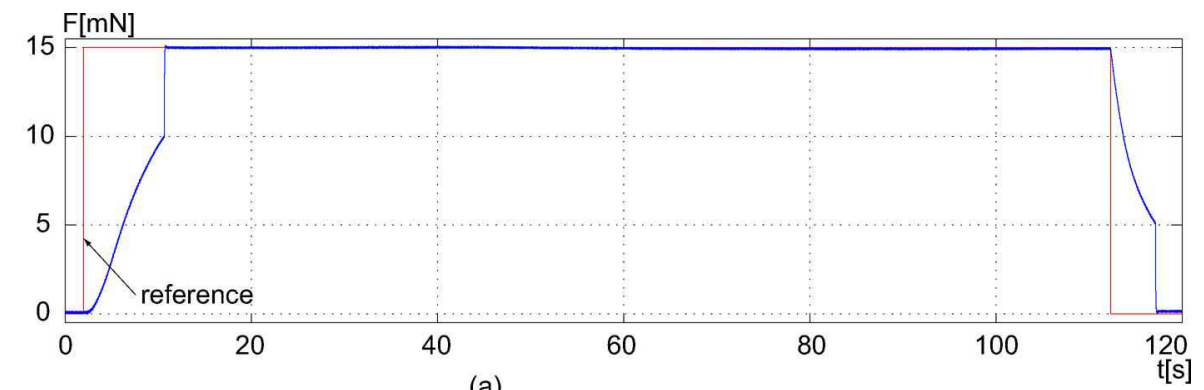

(a)

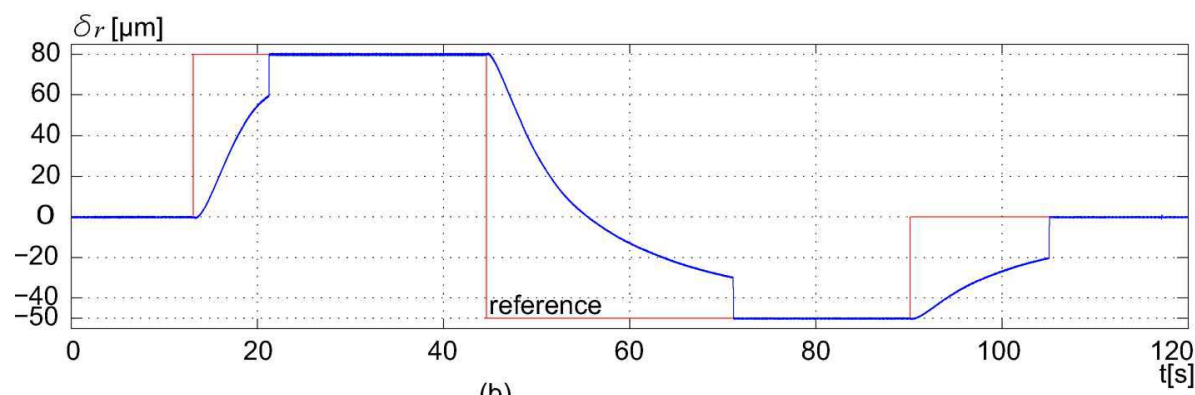

(b)

Fig. 19. Experimental result of an controlled pick-and-place task with controlled position and force: (a) reference and measured output manipulation force (from the left cantilever) and (b) reference and measured output position (from the right cantilever).

and therefore to accelerate the coarse force. When the fine mode starts functioning, the current decreases and becomes constant to hold the last value of the coarse force. Fig. 17 pictures a zoom (with a shifted time scale) of a step response. It indicates first the evolution of the coarse mode, and afterwards the fine mode.

\section{ForCE/POSITION CONTROL OF THE HYBRID MICROGRIPPER}

The two previous sections concern the modeling and control of each cantilever independently. The left cantilever was controlled on force while the right on position. In this section, the microgripper which is composed of the two cantilevers is controlled. For that, the controllers for the position presented in Fig. 8 and for the force presented in Fig. 15 are both implemented. The deflections of the right and of the left cantilevers are reported by using an optical sensor for each one. For the left cantilever, the force is afterwards estimated using the observer.
Fig. 18 shows the experimental setup. The manipulated objects are polystyrene spheres having a diameter less than $1 \mathrm{~mm}$.

To perform a controlled pick-and-place task, the following procedure is followed.

- Initially, the two cantilevers are in close vicinity with the object, i.e., $\delta_{o l}=\delta_{o r}=0$. This condition is only used to facilitate our experiments.

- A step reference of manipulation force $F^{\text {ref }}=15 \mathrm{mN}$ is first applied in order to maintain the object. It corresponds to the picking task.

- When the object is picked, a series of position reference $\delta_{r}^{\text {ref }}=80 \mu \mathrm{m}$ and $\delta_{r}^{\text {ref }}=-50 \mu \mathrm{m}$ is applied.

- Afterwards, the reference position is set to zero.

- Finally, in order to place the object, the reference force is set to zero $F^{\text {ref }}=0 \mathrm{mN}$.

The experimental results are presented in Fig. 19. It clearly shows the efficiency of the controllers used both for the force 
and for the position. At each change of the position (resp. the force) reference, the influence on the force (resp. position) is negligible and is directly rejected.

\section{DISCUSSION}

The experiments were carried out to provide a positioning range up to $80 \mu \mathrm{m}$ and a force range up to $15 \mathrm{mN}$. The static error for both position and force tends towards zero and the accuracy is only dependent on the accuracy of the sensors. Although these performances can already be used for the microassembly of several kinds of objects, for instance, optical micro-objects [28], it is possible to make the microgripper work in higher ranges by applying the necessary input references. Indeed, the $80 \mu \mathrm{m}$ deflection is obtained for a temperature difference of $18^{\circ} \mathrm{C}$ and an external voltage of only $32 \mathrm{~V}$. In fact, the TEC-devices are easily capable to work with $\pm 30 \mathrm{~V}$ allowing a larger stroke. By adjusting the gap between the two cantilevers and by using a more adequate end-effector, it is therefore possible to manipulate smaller objects $(<100 \mu \mathrm{m})$ as it was already done with only piezoelectric microgrippers [8], and then to perform a pick-and-place in large distance relative to their sizes.

As depicted in Fig. 18, the measurement of the displacement as well as of the force requires sensors external to the microgripper and having relatively large dimensions. These characteristics impose a certain space configuration of the sensors in the station and therefore may limit their use in real applications and in packaging aspect. The signals measurement and estimation at the micro and nanoscale constitute emerging topics these last years and several approaches are being done to substitute external and large size sensors.

- Utilization of strain gages. Although these sensors are packageable, they are fragile and the resulting signals are sensitive to noise. Furthermore, for the proposed hybrid microgripper, strain gages may not be suitable because the working temperature of the actuators can destroy or modify the performances of the sensors.

- Utilization of a designed integrated sensors. It consists of designing a sensor additionally to the actuator with the same bulk of material. Generally, electrostatic microgrippers employ this principle. The sensor's parts are often located at the basis of the gripper, whereas the signal to be measured is at its tips. This technique requires a complete modification or a complete redesign of an actuator.

- Utilization of the self-sensing techniques [29]. It consists of using an actuator as a sensor at the same time. Selfsensing techniques are widely used for piezoelectric based microactuators due to the reversibility of the physical principle of these materials. It is particularly a feature for the hybrid microgripper. The influence of the temperature on its performances is under investigation.

During the manipulation of the object, adequate end-effector has to be used in order to avoid the transfer of the temperature variation between the two cantilevers and this object. This end-effector may be made fabricated with a thermal insulating polymer (polyester).

\section{CONCLUSION}

This paper presented the development and the control of a new microgripper that is able to perform both a high range and a high resolution performances. The microgripper is based on a new actuator called hybrid thermo-piezoelectric actuator which combines the thermal bimorph principle and the piezoelectric effect. As the microgripper is made up of two cantilevers, one cantilever is used to position the manipulated object while the second one is used to control the manipulation force. A new control scheme adapted to each hybrid cantilever is proposed. It presents the advantage to automatically switch the coarse and fine positioning or force control. Furthermore, the proposed control scheme has a simplicity of implementation making it very useful for real-time and embedded control systems. Finally, an example of controlled pick-and-place task using the microgripper was carried out. The results clearly show the interest of the developed microgripper and the proposed control scheme for large range, high resolution, and high accuracy micromanipulation or microassembly applications. Future works include the integration of the developed hybrid microgripper inside a micromanipulation/microassembly station where several systems, additionally to the gripper, are cooperatively controlled to perform more complex pick-and-place tasks.

\section{REFERENCES}

[1] M. Rakotondrabe, K. Rabenorosoa, N. Chaillet, and J. Agnus, "Robust feedforward-feedback control of a nonlinear and oscillating 2-dof piezocantilever," IEEE-Trans. Autom. Sci. Eng. (T-ASE), DOI.10.1109/TASE.2010.2099218

[2] D. Jasper, C. Edeler, C. Diederichs, M. Naroska, C. Stolle, and S. Fatikow, "Towards automated robotic nanomanipulation systems," in Proc. IEEE/ASME Int. Conf. Adv. Intell. Mechatron., Singapore, Jul. 14-17, 2009, pp. 94-99.

[3] V. Eichhorn, S. Fatikow, T. Wortmann, C. Stolle, C. Edeler, D. Jasper, Ö. Sardan, P. Bøggild, G. Boetsch, C. Canales, and R. Clavel, "NanoLab: A nanorobotic system for automated pick-and-place handling and characterization of CNTs," in Proc. IEEE Int. Conf. Robot. Autom. (ICRA), Kobe, Japan, May 12-17, 2009, pp. 1826-1831.

[4] R. Casanova, A. Saiz, J. Lacort, J. Brufau, A. Arbat, A. Diéguez, P. Miribel, M. Puig-Vidal, and J. Samitier, "Towards co-operative autonomous $1 \mathrm{~cm} 3$ robots for micro and nanomanipulation applications: MICRON," in Proc. IEEE/RSJ Int. Conf. Intell. Robot. Syst., Edmonton Alberta, Canada, 2005, pp. 760-765.

[5] M. Shahinpoor and K. J. Kim, "Ionic polymer-metal composites: Industrial and medical applications," IOP Smart Material Structures, vol. 14, pp. 197-214, 2005.

[6] M. Kohl, Shape Memory Microactuators. New York: Springer, 2004, ISBN 978-3-540-20635-4.

[7] A. Jain, H. Qu, S. Todd, and H. Xie, "A thermal bimorph micromirror with large bi-directional and vertical actuation," Sens. Actuators A, vol. 9, no. 6, pp. 9-15, 1998.

[8] Y. Haddab, N. Chaillet, and A. Bourjault, "A microgripper using smart piezoelectric actuators," in Proc. IEEE Int. Conf. Intell. Robot. Syst., RSJ IROS, Takamatsu, Japan, 2000, pp. 659-664.

[9] A. Menciassi, A. Eisinberg, G. Scalari, C. Anticoli, M. C. Carrozza, and P. Dario, "Force feedback-based microinstrument for measuring tissue properties and pulse in microsurgery," in Proc. IEEE Int. Conf. Robot. Autom., ICRA, Seoul, Korea, 2001, pp. 626-631.

[10] M. C. Carrozza, A. Eisinberg, A. Menciassi, D. Campolo, S. Micera, and P. Dario, "Towards a force-controlled microgripper for assembling biomedical microdevices," IOP J. Micromech. Microeng. (JMM), vol. 10, pp. 271-276, 2000.

[11] M. Kemper, "Development of a tactile low-cost microgripper with integrated force sensor," in Proc. IEEE Int. Conf. Control Appl., CCA, Taipei, Taiwan, 2004, pp. 1461-1466.

[12] S. Btefisch, V. Seidemann, and S. Bttgenbach, "Novel micro-pneumatic actuator for MEMS," Sens. Actuators A: Phys., vol. 97-98, pp. 638-645, Apr. 1, 2002 
[13] W. Driesen, T. Varidel, S. Rgnier, and J. M. Breguet, "Micro manipulating by adhesion with two collaborating mobile micro robots," $J$. Micromech. Microeng., IOP, vol. 17, pp. s259-s267, 2005.

[14] M. Kohl, E. Just, W. Pfleging, and S. Miyazaki, "SMA microgripper with integrated antagonism," Sens. Actuators A: Phys., vol. 83, no. 1-3, pp. 208-213, May 2000.

[15] F. Beyeler, A. Neild, S. Oberti, D. J. Bell, Y. Sun, J. Dual, and B. J. Nelson, "Monolithically fabricated microgripper with integrated force sensor for manipulating microobjects and biological cells aligned in an ultrasonic field," IEEE J. Micro Electro Mech. Syst. (JMEMS), vol. 16, no. 1, pp. 7-15, Feb. 2007.

[16] C. Yamahata, T. Takekawa, K. Ayano, M. Hosogi, M. Kumemura, B. Legrand, D. Collard, G. Hashiguchi, and H. Fujuta, "Silicon nanotweezers with adjustable and controllable gap for the manipulation and characterization of DNA molecules," in Proc. IEEE Int. Conf. Microtechnol. Med. Biol., MMB, Okinawa, Japan, May 2006, pp. $123-126$.

[17] K. Molhave and O. Hansen, "Electro-thermally actuated microgrippers with integrated force-feedback," IOP J. Micromech. Microeng. (JMM), vol. 15, pp. 1265-1270, 2005.

[18] K. Kim, X. Liu, Y. Zhang, and Y. Sun, "Nanonewton force-controlled manipulation of biological cells using a monolithic MEMS microgripper with two-axis force feedback," IOP J. Micromech. Microeng. (JMM), vol. 18, p. 055013, 2008.

[19] G. Greitman and R. A. Buser, "Tactile microgripper for automated handling of microparts," Sens. Actuators A: Phys., vol. 53, no. 1-3, pp. 410-415, May 1996.

[20] M. Rakotondrabe and I. A. Ivan, "Principle, characterization and control of a new hybrid thermo-piezoelectric microactuator," in Proc. IEEE Int. Conf. Robot. Autom., ICRA, Anchorage Alaska, USA, May 2010, accepted and to appear in the proceeding.

[21] Y. Haddab, "Design and development of a micromanipulation system with force/position control," (in French) Ph.D. dissertation, FrancheComté Univ., Besançon, France, 2000.

[22] M. Rakotondrabe, C. Clévy, and P. Lutz, "Modelling and robust position/force control of a piezoelectric microgripper," in Proc. IEEE Int. Conf. Autom. Sci. Eng. (CASE), Scottsdale, AZ, Sep. 2007, pp. 39-44.

[23] J. G. Smits and W.-S. Choi, "The constituent equations of piezoelectric heterogeneous bimorph," in Proc. IEEE Ultrasonics Symp., 1990, pp. $1275-1278$.

[24] M. Rakotondrabe, C. Clévy, and P. Lutz, "Complete open loop control of hysteretic, creeped and oscillating piezoelectric cantilevers," IEEE Trans. Autom. Sci. Eng. (TASE), vol. 7, no. 3, pp. 440-450, Jul. 2010.

[25] J. G. Smits and W.-S. Choi, "Equations of state including the thermal domain piezoelectric and pyroelectric heterogeneous bimorphs," in Proc. IEEE Ultrasonics Symposium, 1992, pp. 1035-1038.

[26] M. Rakotondrabe, Y. Haddab, and P. Lutz, "Nonlinear modeling and estimation of force in a piezoelectric cantilever," in Proc. IEEE/ASME Int. Conf. Adv. Intell. Mechatron., AIM, Zurich, Switzerland, Sep. 2007, pp. 1-6.
[27] S. Bargiel, K. Rabenorosoa, C. Clevy, C. Gorecki, and P. Lutz, "Towards micro-assembly of hybrid MOEMS components on a reconfigurable silicon free-space micro-optical bench," J. Micromech. Microeng., vol. 20, no. 4, Mar. 2010, doi:10.1088/0960-1317/20/4/ 045012.

[28] S. Bargiel, K. Rabenorosoa, C. Clevy, C. Gorecki, and P. Lutz, "Towards micro-assembly of hybrid MOEMS components on a reconfigurable silicon free-space micro-optical bench," J. Micromech. Microeng., vol. 20, no. 4, Mar. 2010.

[29] M. Rakotondrabe, I. A. Ivan, S. Khadraoui, C. Clévy, P. Lutz, and N. Chaillet, "Dynamic displacement self-sensing and robust control of cantilevered piezoelectric actuators dedicated to microassembly tasks," in Proc. IEEE/ASME Int. Conf. Intell. Mater., AIM, Montreal, Canada, Jul. 2010, pp. 557-562.

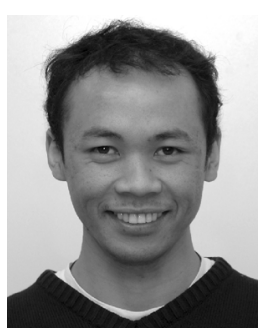

Micky Rakotondrabe (S'05-M'07) received the Engineering diploma from the ISTN-ICAM, Lille, France, in 2002, the DEA diploma (M.Sc.) in automatic control from the INSA, Lyon, France, in 2003, and the Ph.D. degree in automatic control from the Université de Franche-Comté, Besançon, France, in 2006.

Since 2007, he has been an Associate Professor with the Université de franche-Comté and a Researcher with the FEMTO-ST Institute. His field of research concerns the control theory applied to microsystems and microrobots, including modeling, signal estimation/observation, identification, and (feedback and feedforward) control.

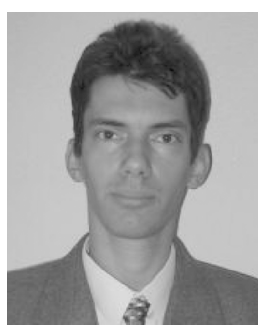

Ioan Alexandru Ivan (M'10) has been working towards the Ph.D. degree in microelectronics at the University of Besançon, Besançon, France, since 2006.

He is a Research Fellow at the French institute FEMTO-ST, Besançon, France, from 2008 to 2010, under the FP7 Intra-European Marie Curie project called MicroPAdS. In parallel, he is a Lecturer in Electrical Engineering at Valahia University, Romania. His background is a Technological Physics Engineer at the University of Bucharest, Bucharest, Romania, since 1999. His actual research interests are mainly in piezoelectric microsystems for micro and nanoscale characterization, manipulation, and control. 